\title{
Role of miR-145 in chronic constriction injury in rats
}

\author{
XIAOLIN PANG $^{1}$, YUANZHANG TANG ${ }^{2}$ and DONGYA ZHANG ${ }^{1}$ \\ ${ }^{1}$ Department of Anesthesiology, First Hospital of Tsinghua University, Beijing 100016; \\ ${ }^{2}$ Department of Pain Management, Xuanwu Hospital of Capital Medical University, Beijing 100053, P.R. China
}

Received June 9, 2015; Accepted June 7, 2016

DOI: $10.3892 /$ etm.2016.3900

\begin{abstract}
The present study aims to investigate the effects and underlying mechanisms of miRNA-145 (miR-145) in rat models of chronic constriction injury (CCI). Rats were randomly divided into control, sham, CCI, agomiRNA (agomiR)-normal control (NC) and agomiR-145 groups ( $\mathrm{n}=25$ in each group); in addition, 30 rats with CCI were divided into small hairpin (sh)RNA-NC and shRNA-ras responsive element binding protein 1 (RREB1) groups. Paw withdrawal thermal latency (PWTL) and paw withdrawal mechanical threshold (PWMT) were detected. Reverse transcription-quantitative polymerase chain reaction was used to detect miR-145 expression levels, and western blotting was performed to measure RREB1 and phosphorylated-protein kinase B (p-AKT) expression levels. In addition, a dual luciferase reporter assay was conducted to identify the target gene of miR-145. PWMT and PWTL were decreased in CCI rats and this decrease was alleviated by miR-145 injection. At 1, 3, 5 and 7 days after CCI, miR-145 expression level in the spinal cord tissue of rats in the CCI group was significantly decreased compared with 1 day before CCI $(\mathrm{P}<0.05)$. Compared with the CCI group, miR-145 expression level in the agomiR-145 group was significantly higher $(\mathrm{P}<0.05)$. In addition, expression levels of RREB1 and p-AKT were significantly increased in the CCI group and significantly decreased in the agomiR-145 group $(\mathrm{P}<0.05)$. Furthermore, knockdown of RREB1 expression by shRNA-RREB1 significantly increased values of PWMT and PWTL, decreased expression levels of RREB1 and p-AKT, and increased miR-145 expression levels $(\mathrm{P}<0.05)$. Further investigation demonstrated that miR-145 can bind with RREB1 mRNA. In conclusion, miR-145 may be involved in the development of CCI through regulating the expression of RREB1.
\end{abstract}

Correspondence to: Dr Dongya Zhang, Department of Anesthesiology, First Hospital of Tsinghua University, 2 First Alley, Jiuxianqiao Road, Beijing 100016, P.R. China

E-mail: zdy000@126.com

Key words: microRNA-145, chronic constriction injury, ras responsive element binding protein 1, phosphorylated protein kinase B

\section{Introduction}

Neuropathic pain is a common chronic pain, and includes spontaneous pain, allodynia, hyperalgesia and hypersensitivity, and pain disorders (1). Clinically, sciatica and chronic back pain are the most common types of neuropathic pain, and the pathogenesis is complicated (2). The primary causes are the degeneration of the corresponding organs, lumbar disc herniation caused by trauma, nerve damage, calcification of ligaments, bone hyperplasia, inflammation and tumors (3-5). Due to the long period of clinical treatment, disease recurrence and poor prognosis, the quality of life of patients with neuropathic pain is greatly affected. Therefore, it is important to investigate the mechanisms underlying neuropathic pain at molecular level.

miRNA are a type of non-coding RNA molecules (18-22 nt in length) in eukaryotes, which can regulate the expression of proteins at the mRNA level (6-8). Studies have shown that miRNA molecules serve important biological functions in the development, apoptosis and synaptic release of the nervous system (9-11). miR-145 is an important miRNA that is associated with numerous diseases, including hypertension, cancer and inflammation. For example, miR-145 regulates the effect of aspirin on endothelial cell proliferation and inflammation (12). Moreover, miR-145 is reported to be closely associated with airway smooth muscle inflammation in chronic obstructive pulmonary disease (13). In addition, it has been demonstrated that the expression of miRNA-145 (miR-145) is significantly decreased in damaged nerve tissues $(14,15)$, suggesting that miR-145 may serve an important role in neuropathic pain caused by nerve damage. Ras responsive element binding protein 1 (RREB1) is an important transcription factor, which can initiate the transcription of many downstream genes once activated. It has been reported that RREB1 is associated with type 2 diabetes and tumor $(16,17)$. However, the expression pattern and role of RREB1 in neuropathic pain remains unknown.

At present, the expression of miR-145 and its function in chronic constriction sciatica (CCI), one type of neuropathic pain, is unclear. In the present study, the role of miR-145 in CCI is investigated using a CCI model in rats. Intrathecal injection of agomiRNA (agomiR)-145 and in vivo transfection of small hairpin (sh) RNA-ras responsive element binding protein 1 (RREB1) was performed, and the pain behaviors of PWMT and PWTL were measured. In addition, the expression levels of miR-145, RREB1 and phosphorylated protein kinase B (p-AKT) are detected. 


\section{Materials and methods}

Animals. A total of 155 healthy Sprague-Dawley (SD) rats (specific-pathogen-free; weight, 200-250 g; male) were purchased from Chengdu Dashuo Biotech Co. (Chengdu, China). The mice were maintained in standard conditions at room temperature with $50 \%$ humidity, under $12 \mathrm{~h} / 12 \mathrm{~h}$ light/dark cycle and with free access to food and water. All animal experiments were conducted according to the ethical guidelines of the First Hospital of Tsinghua University (Beijing, China).

Reagents. TRIzol Total RNA Extraction kit was purchased from Invitrogen (Thermo Fisher Scientific, Inc., Waltham, MA, USA). PrimeScript RT Reagent kit and SYBR PrimeScript RT-PCR kit were purchased from Takara Biotechnology Co., Ltd. (Dalian, China). The plasmids of shRNA-normal control (NC), shRNA-RREB1, agomiR-NC and agomiR-145 were purchased from Guangzhou RiboBio Co., Ltd. (Guangzhou, China). EntransterTM-in vivo transfection reagent was purchased from Engreen Biosystem Co., Ltd. (Beijing, China). Goat anti-rat RREB1 (St. Louis Park, MN, USA), rabbit anti-rat p-AKT (Cell Signaling Technology, Inc., Danvers, MA, USA), mouse anti-rat GAPDH and horseradish peroxidase (HRP)-conjugated secondary antibodies were purchased from Abcam (Burlingame, CA, USA). Dual Luciferase Reporter Assay kit was purchased from Promega Corporation (Madison, WI, USA).

Establishment of CCI model and animal grouping. A total of 125 rats were randomly divided into the control, sham, CCI model, agomiR-145 and agomiR-NC groups, with 25 rats in each group. For the CCI group, the left sciatic nerve of rats was exposed by surgery and ligated. For the sham group, the left sciatic nerve of rats was exposed by surgery, but without ligation. Following the establishment of the CCI model, intrathecal injection of agomiR-145 was performed in the agomiR-145 group once daily for 7 consecutive days. For the agomiR-NC group, intrathecal injection of agomiR-NC was performed once daily for 7 consecutive days. For the control group, no surgery or treatment was performed.

Following the establishment of the CCI model, an additional 30 healthy male SD rats were randomly divided into shRNA-NC and shRNA-RREB1 groups. In vivo transfection of shRNA-RREB1 and shRNA-NC plasmids was performed by EntransterTM-in vivo transfection reagent, according to the manufacturer's instructions. The transfection was performed once daily for 7 consecutive days following the establishment of CCI.

At 1 day prior to, and 1,3,5 and 7 days following surgery, the PWTL and PWMT of rats in each group were detected. At each time point, 5 rats in each group were used. In addition, at each time point, 5 rats in each group were sacrificed by cervical dislocation following anesthesia using $1 \%$ pentobarbital (EMD Millipore, Billerica, MA, USA). The lumbar spinal cord tissues were collected from these rats for miRNA and protein detection.

PWTL. Rats were placed on a 7370 Planter Test machine (Ugo Basile S.R.L., Varese, Italy) and allowed to acclimatize for 3 days prior to testing. A radiant thermal stimulator was focused onto the plantar surface of the hind paw for $15 \mathrm{sec}$. The nociceptive endpoints in the radiant heat test were the characteristic lifting or licking of the hind paw, and the time to the endpoint was recorded as the PWTL. To avoid tissue damage, a cut-off time of $30 \mathrm{sec}$ was used. There were 3 repeats of the trial per rat, and 5 min intervals between trials. The mean PWTL was obtained from the three recordings.

PWMT. A 2390 Electronic von-Frey Anesthesiometer (Naturegene Life Science, Cranbury, NJ, USA) was used. PWMT was performed according to the method described by Hargreaves (18). Brisk withdrawal or paw flinching were considered as positive responses. There were 3 repeats of the trial per rat, and 5 min intervals between trials. The mean PWMT was calculated from the three recordings.

Reverse transcription-quantitative polymerase chain reaction (RT- $q P C R)$. Total RNA was extracted from lumbar spinal cord tissues using the TRIzol Total RNA Extraction kit, according to the manufacturer's instructions. RNA was reverse transcribed into cDNA using a PrimeScript RT Reagent kit. The expression level of miR-145 was detected by RT-qPCR. The forward primer sequence for miR-145 was 5'-CCAGTTTTCCCAGGAATC-3'; the reverse primer sequence was a universal primer provided by the kit. RT-qPCR was performed using a SYBR Green PrimeScript RT-PCR kit, according to the manufacturer's instructions. The volume of the PCR reaction system was $20 \mu \mathrm{l}$, which consisted of $10 \mu \mathrm{l}$ qRT-PCR mix, $0.5 \mu \mathrm{l}$ forward primer and $0.5 \mu \mathrm{l}$ reverse

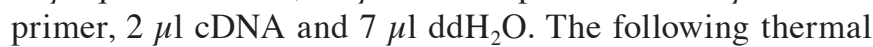
cycling conditions were used: Pre-denaturation at $95^{\circ} \mathrm{C}$ for $1 \mathrm{~min} ; 40$ cycles of $95^{\circ} \mathrm{C}$ for $15 \mathrm{sec}$; and $60^{\circ} \mathrm{C}$ for $30 \mathrm{sec}$. The $2^{-\Delta \Delta \mathrm{Ct}}$ method was used to calculate the relative expression levels of miR-145 (19).

Western blotting analysis. Tissues were ground into a powder in liquid nitrogen, and were lysed on ice in radioimmunoprecipitation assay buffer (RIPA) lysis buffer (Beyotime Institute of Biotechnology, Beijing, China) with $1 \mathrm{mM}$ PMSF for $30 \mathrm{~min}$. Total proteins were extracted by centrifugation at $14,000 \times g$ for $10 \mathrm{~min}$ at $4^{\circ} \mathrm{C}$. Total proteins were extracted $(15 \mu \mathrm{g})$ from lumbar spinal cord tissues and separated by a $10 \%$ SDS-PAGE. Next, proteins were transferred onto a polyvinylidene fluoride membrane. After blocking with non-fat milk, the membrane was incubated with the following rabbit primary antibodies at $4^{\circ} \mathrm{C}$ overnight: Anti-RREB1 (ab64168, 1:2,000), anti-p-AKT (ab38449, 1:2,000) and anti-GAPDH (ab8245, 1:5,000, all Abcam). After washing, the membrane using $0.1 \%$ phosphate-buffered saline and Tween 20 for 5 mins each time and in total three times, the membrane was incubated with HRP-conjugated secondary antibodies [goat anti-rabbit IgG (ab6721), and goat anti-mouse IgG H\&L (ab6789, both 1:10,000, both Abcam)] at room temperature for $1 \mathrm{~h}$. Finally, the membrane was developed by enhanced chemiluminescence plus reagent (Hanbio Biotechnology Co. Ltd., Shanghai, China). The developed film was scanned using AlphaImager gel imaging systems (AlphaImager, Santa Clara, CUA, USA), and the western blot images were analyzed using Quantity One software 


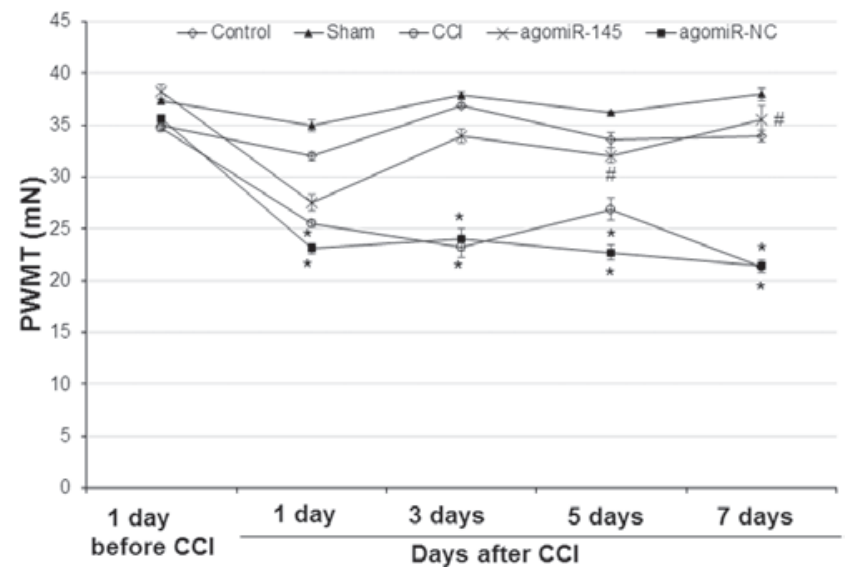

Figure 1. Analysis of PWMT in CCI rat models. Rats were randomly divided into control, sham, CCI, agomiR-145 and agomiR-NC groups (n=25 in each group). PWMT was performed 1 day before and $1,3,5$ and 7 days after $\mathrm{CCI} .{ }^{*} \mathrm{P}<0.05$ vs. PWMT 1 day before CCI; ${ }^{*} \mathrm{P}<0.05$ vs. the CCI group. The error bars show the mean \pm standard deviation. PWMT, paw withdrawal mechanical threshold; CCI, chronic constriction injury; NC, normal control.

version 4.62 (Bio-Rad Laboratories, Inc., Hercules, CA, USA). GAPDH was used as an internal control.

Dual luciferase reporter assay. The dual luciferase reporter assay was performed using a Dual Luciferase Reporter Assay kit. Briefly, the wild type and mutant 3'-untranslated region (UTR) of RREB1 mRNA were cloned into a pMIR-REPORT vector. Then, these plasmids, together with miR-145 mimics (100 nM), were co-transfected into 293T cells (Cell Bank of the Chinese Academy of Sciences, Shanghai, China). After incubation at $37^{\circ} \mathrm{C}$ for $24 \mathrm{~h}$, cells were lysed by washing with cold PBS, the cells were incubated with RIPA lysis buffer with $1 \%$ PMSF on ice for $20 \mathrm{~min}$ and fluorescence was detected using GloMax 20/20 Luminometer (Promega Corporation). Cells without miR-145 mimic transfection were used as control cells. The fluorescence intensity of Renilla was used as an internal reference.

Statistical analysis. Data was expressed as the mean \pm standard deviation and was analyzed using SPSS statistical analysis software (version 17.0; SPSS, Inc., Chicago, IL, USA). A paired t-test was used to analyze the difference between groups. $\mathrm{P}<0.05$ was considered to indicate a statistically significant difference.

\section{Results}

Effect of miR-145 on PWMT in CCI rat models. To investigate the effect of miR-145 on the mechanical threshold of rats, PWMT was performed 1 day prior to, and 1, 3, 5 and 7 days after CCI. As shown in Fig. 1, at 1 day prior to CCI, there was no significant difference in mechanical threshold among the 5 groups. In the control and sham group, the mechanical threshold was not significantly different at any time points. However, the mechanical threshold in the CCI and agomiR-NC group at 1, 3, 5 and 7 days after CCI was significantly decreased compared with 1 day prior to $\mathrm{CCI}$ (CCI: 1 day, $\mathrm{P}=0.034 ; 3$ day, $\mathrm{P}=0.024 ; 5$ day, $\mathrm{P}=0.042 ; 7$ day, $\mathrm{P}=0.020$ and agomiR-NC: 1 day, $\mathrm{P}=0.023 ; 3$ day, $\mathrm{P}=0.029$; 5 day, $\mathrm{P}=0.022 ; 7$ day, $\mathrm{P}=0.019)$. At 5 and 7 days after $\mathrm{CCI}$, the mechanical threshold in the agomiR-145 group was significantly increased compared with the CCI group (5 day, $\mathrm{P}=0.033 ; 7$ day, $\mathrm{P}=0.027)$. No significant difference was identified between the CCI and agomiR-NC groups at any time points. These results suggest that miR-145 treatment alleviates the mechanical threshold decrease induced by CCI.

Effect of miR-145 on PWMT in CCI rat models. To determine the effect of miR-145 on thermal latency, PWTL was performed 1 day prior to, and 1, 3, 5 and 7 days after CCI. Similar to PWMT, no significant difference was found 1 day prior to CCI among the 5 groups (Fig. 2). In the control and sham group, there was no significant difference in thermal latency at any time points. However, at 1,3,5 and 7 days after $\mathrm{CCI}$, the thermal latency in the CCI and agomiR-NC groups was significantly lower compared with the thermal latency 1 day prior to CCI (CCI: 1 day, $\mathrm{P}=0.036 ; 3$ day, $\mathrm{P}=0.023$; 5 day, $\mathrm{P}=0.040 ; 7$ day, $\mathrm{P}=0.022$ and agomiR-NC: 1 day, $\mathrm{P}=0.032 ; 3$ day, $\mathrm{P}=0.027 ; 5$ day, $\mathrm{P}=0.037 ; 7$ day, $\mathrm{P}=0.025$ ). After miR-145 treatment, the thermal latency was elevated in the agomiR-145 group. Compared with the CCI group, the thermal latency in the agomiR-145 group was significantly higher at 3, 5 and 7 days after CCI ( 3 day, $\mathrm{P}=0.039$; 5 day, $\mathrm{P}=0.027 ; 7$ day, $\mathrm{P}=0.017)$. There was no significant difference in the thermal latency between the CCI and agomiR-NC groups at any time points. This data indicates that miR-145 treatment improves the thermal latency decrease induced by CCI.

Expression of miR-145 in CCI rat models. To detect the expression of miR-145 in lumbar spinal cord tissues, RT-qPCR was conducted 1 day prior to, and 1, 3, 5 and 7 days after CCI. Results are presented in Fig. 3. There was no significant difference in miR-145 expression levels between the control and sham groups, or between the CCI and agomiR-NC groups, at any time points. Compared with the control group, miR-145 expression levels in the CCI group was significantly lower at 1, 3, 5 and 7 days after CCI (1 day, $\mathrm{P}=0.043 ; 3$ day. $\mathrm{P}=0.038 ; 5$ day, $\mathrm{P}=0.002 ; 7$ day, $\mathrm{P}=0.001)$. After treatment with agomiR-145, miR-145 expression was increased in the agomiR-145 group. The expression level of miR-145 1, 3, 5 and 7 days after CCI was $2.18 \pm 0.43,5.34 \pm 0.57,6.18 \pm 0.81$ 


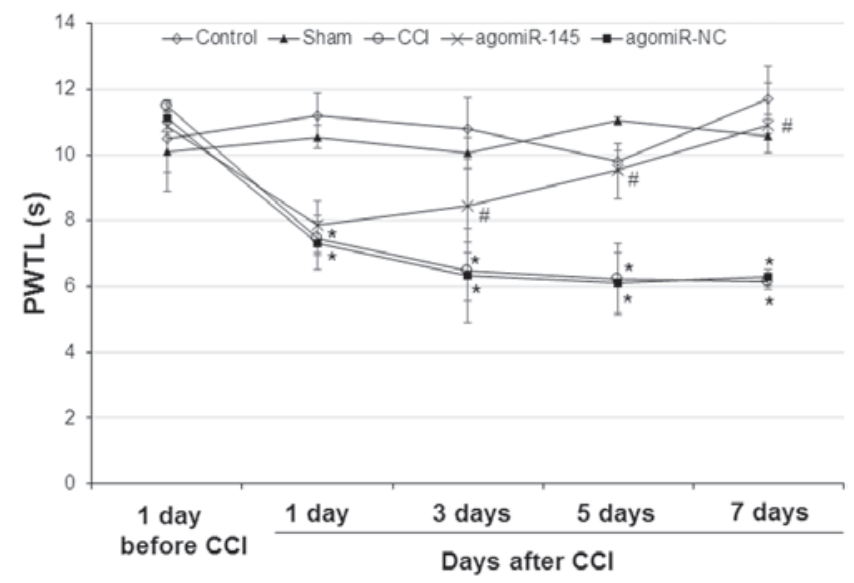

Figure 2. Analysis of PWTL in CCI rats. Rats were randomly divided into control, sham, CCI, agomiR-145 and agomiR-NC groups (n=25 in each group). PWTL was performed 1 day before and 1,3, 5 and 7 days after CCI. " $\mathrm{P}<0.05$ vs. PWTL 1 day before CCI; ${ }^{*} \mathrm{P}<0.05$ vs. the CCI group. The error bars show the mean \pm standard deviation. PWMT, paw withdrawal mechanical threshold; CCI, chronic constriction injury; $\mathrm{NC}$, normal control.

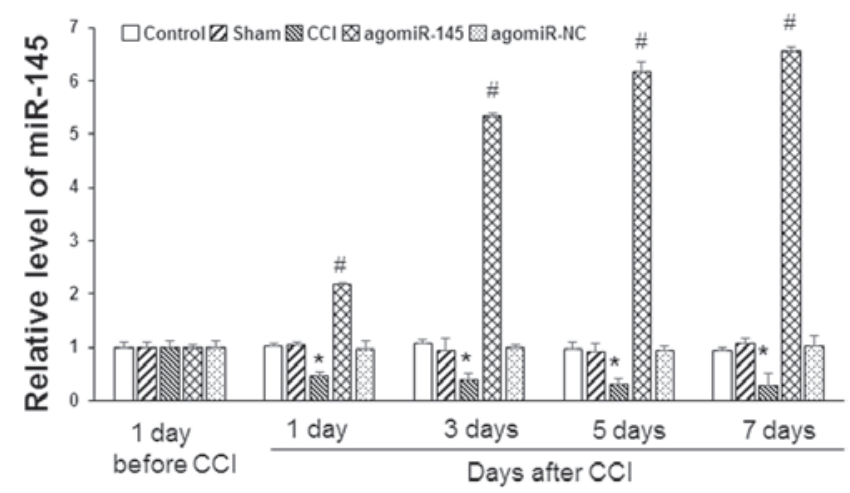

Figure 3. Analysis of miR-145 expression in lumbar spinal cord tissue. Rats were randomly divided into control group, sham, CCI, agomiR-145 and agomiR-NC groups ( $\mathrm{n}=25$ in each group). At 1 day before and 1,3,5 and 7 days after CCI, lumbar spinal cord tissues were collected from 5 rats in each group. The expression level of miR-145 in spinal cord tissue was detected using reverse transcription-quantitative polymerase chain reaction. "P $<0.05$ vs. the control group; ${ }^{\text {"P}} \mathrm{P}<0.05$ vs. the CCI group. The error bars show the mean \pm standard deviation. CCI, chronic constriction injury; NC, normal control.

and $6.56 \pm 1.16$, respectively, in the agomiR-145 group, which was significantly higher compared with the CCI group (1 day, $\mathrm{P}=0.027 ; 3$ day, $\mathrm{P}=0.007 ; 5$ day, $\mathrm{P}=0.001 ; 7$ day, $\mathrm{P}=0.001$ ). Thus, the results demonstrate that miR-145 expression is decreased in CCI rats, and that agomiR-145 treatment can increase miR-145 expression levels in CCI rats.

Effect of miR-145 on the expression level of RREBI and $p$-AKT in CCI rat models. To measure the expression of RREB1 and p-AKT in the lumbar spinal cord tissue of CCI rats 1 day prior to, and 1,3,5 and 7 days after $\mathrm{CCI}$, western blot analysis was performed; representative and quantitative western blot results are presented in Fig. 4. In the control group, there was no significant difference in the expression of RREB1 or p-AKT at any time points (Fig. 4A). In the CCI group, the expression levels of RREB1 and p-AKT were elevated (Fig. 4B). As shown in Fig. 4C, following treatment with agomiR-145, the expression levels of RREB1 and p-AKT in the agomiR-145 group were decreased at 3 , 5 and 7 days after CCI. Statistically, the expression levels of RREB1 ( 1 day; $\mathrm{P}=0.039 ; 3$ day, $\mathrm{P}=0.021 ; 5$ day, $\mathrm{P}=0.027$; 7 day, $\mathrm{P}=0.036$; Fig. 4D) and $\mathrm{p}-\mathrm{AKT}(1 \mathrm{day} ; \mathrm{P}=0.031 ; 3$ day, $\mathrm{P}=0.027 ; 5$ day, $\mathrm{P}=0.021 ; 7$ day, $\mathrm{P}=0.033$; Fig. 4E) in the $\mathrm{CCI}$ group were significantly higher compared with the control group at 1, 3, 5 and 7 days after CCI. In addition, at 1, 3, 5 and 7 days after CCI, the agomiR-145 group had significantly lower expression levels of RREB1 ( 1 day; $\mathrm{P}=0.048$; 3 day, $\mathrm{P}=0.032 ; 5$ day, $\mathrm{P}=0.015 ; 7$ day, $\mathrm{P}=0.018$; Fig. 4D) and p-AKT ( 1 day; $\mathrm{P}=0.046 ; 3$ day, $\mathrm{P}=0.016 ; 5$ day, $\mathrm{P}=0.011$; 7 day, $\mathrm{P}=0.023$; Fig. 4E) compared with the CCI group. The changes in expression levels of RREB1 and p-AKT in the sham and agomiR-NC groups were similar to those in the control and CCI groups, respectively (data not shown). These results indicate that $\mathrm{CCI}$ increases the expression levels of RREB1 and p-AKT in lumbar spinal cord tissues, whereas miR-145 decreases the increase induced by CCI.

Effect of RREB1 protein on pain behavior, protein expression and miR-145 expression levels in CCI rat models. To determine the effect of RREB1 in CCI rat models, in vivo transfection of shRNA-RREB1 and shRNA-NC plasmids was performed following the establishment of $\mathrm{CCI}$ in rats. The pain behavior of PWMT and PWTL, protein expression of RREB1 and p-AKT, and expression level of miR-145 were detected. As shown in Fig. 5A, the mechanical threshold of CCI rats in the shRNA-NC group 1,3,5 and 7 days after CCI was 
A

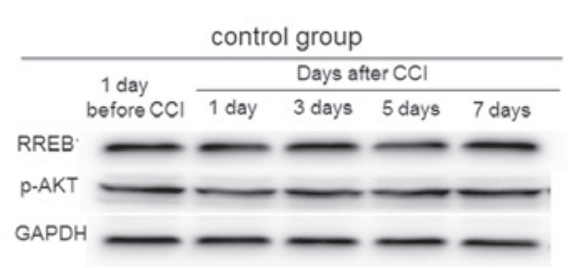

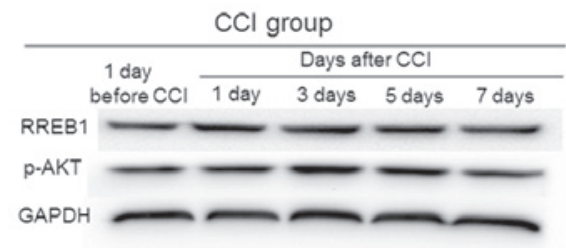

C

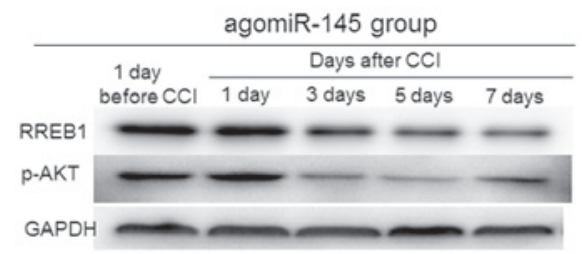

D

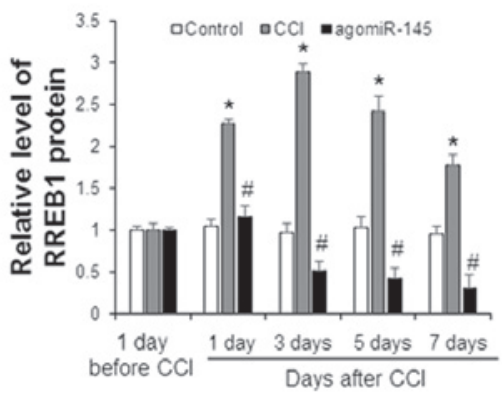

$\mathbf{E}$

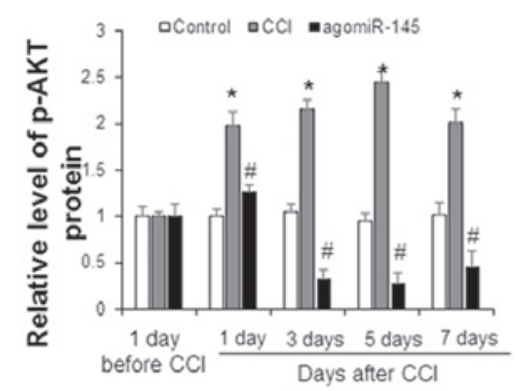

Figure 4. Analysis of RREB1 and p-AKT expression in lumbar spinal cord tissue. At 1 day before and 1,3,5 and 7 days after CCI, lumbar spinal cord tissues were collected from 5 rats in each group. Expression levels of RREB1 and p-AKT in spinal cord tissue were measured using western blotting analysis (A) Representative western blotting results showing expression levels of RREB1 and p-AKT in the (A) control, (B) CCI and (C) agomiR-145 groups. Relative expression levels of (D) RREB1 and (E) p-AKT. "P $<0.05$ vs. the control group; ${ }^{*} \mathrm{P}<0.05$ vs. the CCI group. The error bars show the mean \pm standard deviation. RREB1, ras responsive element binding protein 1; p-AKT, phosphorylated protein kinase B; CCI, chronic constriction injury; NC, normal control.

A

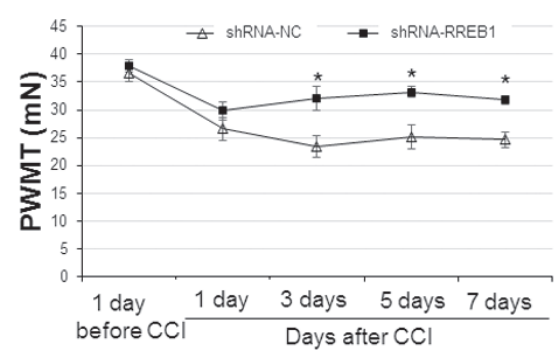

C

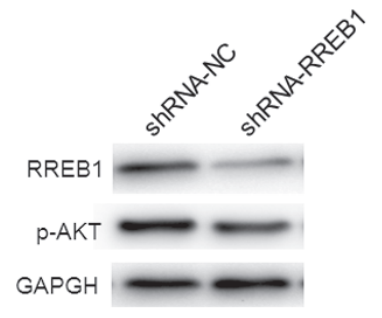

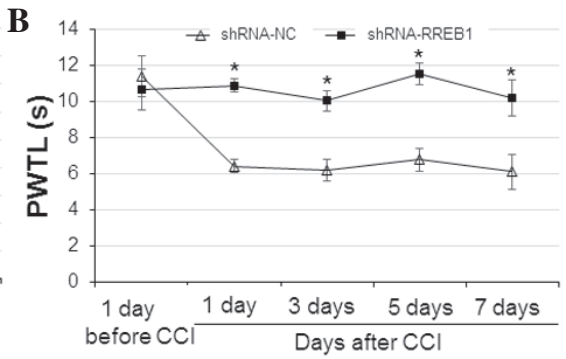

D

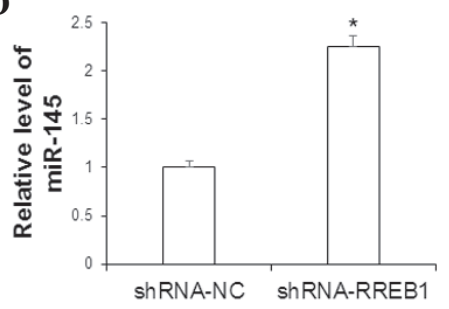

Figure 5. Analysis of pain behavior, protein expression and miR-145 expression level in CCI rats. Rats with CCI were divided into shRNA-RREB1 and shRNA-NC groups ( $n=15$ rats in each group). Lumbar spinal cord tissues were collected 7 days after CCI. (A) PWMT and (B) PWTL was performed 1 day before and 1,3,5 and 7 days after CCI. (C) Expression levels of RREB1 and p-AKT were detected by western blotting analysis. (D) Expression level of miR-145 was detected by reverse transcription-quantitative polymerase chain reaction. ${ }^{*} \mathrm{P}<0.05$ vs. the shRNA-NC group. The error bars show the mean \pm standard deviation. miR-145, microRNA-145; CCI, chronic constriction injury; shRNA, small hairpin RNA; RREB1, ras responsive element binding protein 1; NC, normal control; PWMT, paw withdrawal mechanical threshold; PWTL, paw withdrawal thermal latency; p-AKT, phosphorylated protein kinase B.

decreased compared with that at 1 day prior to CCI. However, in the shRNA-RREB1 group, the mechanical threshold of CCI rats was increased compared with the shRNA-NC group; this difference was significant at 3,5 and 7 days 
A

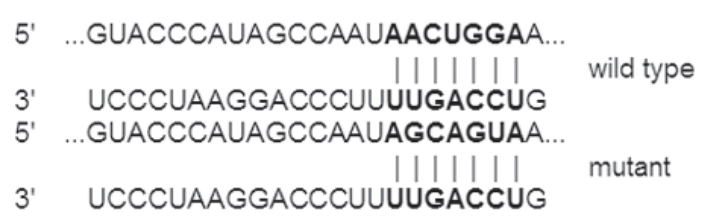

B

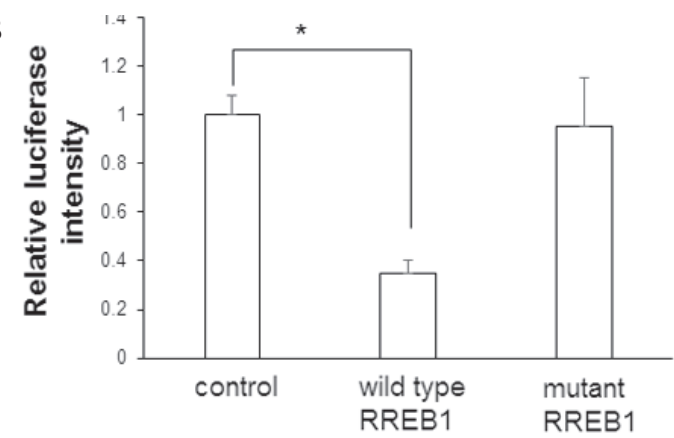

Figure 6. Analysis of miR-145 binding with RREB1 mRNA. (A) Sequences of the wild type and mutant 3'-UTR of RREB1 mRNA. (B) Dual luciferase reporter assay was performed to detect the binding of miR-145 with RREB1 mRNA. 293T cells were transfected with miR-145 mimics and wild type or mutant RREB1. Cells without miR-145 mimic transfection were used as the control. " $\mathrm{P}<0.05$ vs. the control. The error bars show the mean \pm standard deviation. miRNA, microRNA; RREB1, ras responsive element binding protein 1; UTR, untranslated region.

after $\mathrm{CCI}$ ( 3 day, $\mathrm{P}=0.032 ; 5$ day, $\mathrm{P}=0.370 ; 7$ day, $\mathrm{P}=0.041$ ). Similar results were observed in the thermal latency of $\mathrm{CCI}$ rats (Fig. 5B). Thermal latency in the shRNA-RREB1 group was significantly increased compared with the shRNA-NC group $1,3,5$ and 7 days after $\mathrm{CCI}(1$ day, $\mathrm{P}=0.021 ; 3$ day, $\mathrm{P}=0.026 ; 5$ day, $\mathrm{P}=0.031 ; 7$ day, $\mathrm{P}=0.043)$. As shown in Fig. 5C, the expression levels of RREB1 and p-AKT 7 days after CCI were decreased following shRNA-RREB1 transfection. In addition, compared with the shRNA-NC group, the shRNA-RREB1 group had significantly higher levels of miR-145 7 days after CCI ( $\mathrm{P}=0.023$; Fig. 5D). Collectively, these results suggest that downregulation of RREB1 protein expression levels in CCI rats may improve pain behavior, decrease p-AKT protein expression and increase miR-145 expression levels.

Effect of miR-145 on RREB1. To determine whether RREB1 mRNA is one of the targets of miR-145, a dual luciferase reporter assay was performed following transfection of miR-145 mimics and wild type or mutant RREB1 mRNA. Cells without miR-145 mimic transfection were used as control cells. The sequences of the wild type and mutant 3'-UTR region of RREB1 mRNA are presented in Fig. 6A. The luciferase intensity is presented in Fig. 6B. In cells transfected with miR-145 mimics and wild type RREB1, the luciferase intensity was significantly decreased compared with control cells $(\mathrm{P}=0.017)$. However, there was no significant difference in luciferase intensity between control cells and cells transfected with miR-145 mimics and mutant RREB1. This data suggests that RREB1 mRNA is a target of miR-145.

\section{Discussion}

Due to the lack of effective treatment methods, neuropathic pain severely threatens the health of patients (20). At present, the molecular mechanisms underlying neuropathic pain are unclear. In addition, effective diagnosis and treatment targets for neuropathic pain are lacking. It is reported that miRNA molecules are important regulatory factors in the nervous system and are extensively involved in various physiological and pathological activities of the nervous system, such as memory, neural remodeling and neurodegenerative changes (21).
In the present study, the role of miR-145 in neuropathic pain is investigated in CCI rat models. The values of PWMT and PWTL in the CCI group were significantly decreased, suggesting that the CCI model was successfully established in rats. Consistent with the results presented by Zhang et al (22), miR-145 expression levels in spinal cord tissue were significantly decreased in CCI rats. To further elucidate the role of miR-145 in CCI development, intrathecal injection of agomiR-145 was performed. The results demonstrate that the values of PWMT and PWTL in the agomiR-145 group were significantly higher compared with the CCI group. This indicates that miR-145 improves pain behaviors of CCI rats and may serve a role in the development of CCI. Bioinformatical analysis using the online software called Targetscan (www.targetscan.com.cn) was used for prediction, and it demonstrates that agomiR-145 may regulate the expression of the transcription factor RREB1. However, Kent et al (23) observed that RREB1 can inhibit the expression of miR-145. In addition, studies have demonstrated that miR-145 can inhibit the phosphatidylinositol 3-kinase (PI3K)/AKT signaling pathway through regulating the expression of integrin-linked kinase $(24,25)$.

The PI3K/AKT signaling pathway serves an important role in vascular endothelial growth factor (VEGF)-induced hyperalgesia (26). VEGF is involved in the occurrence of neuropathic pain by activating the PI3K/AKT signaling pathway in neurons, and inducing the expression of transient receptor potential vanilloid subfamily member 1 (25). In the current study, the protein expression levels of RREB1 and p-AKT were examined in spinal cord tissue of rats. It was identified that the protein expression levels of RREB1 and p-AKT were significantly increased in CCI rats. However, following intrathecal injection of agomiR-145, the protein expression levels of RREB1 and p-AKT were significantly decreased. In order to further determine the association between miR-145 and RREB1, RREB1 expression was interfered by in vivo transfection of shRNA-RREB1. Following the downregulation of RREB1 expression, PWTL and PWMT values in CCI rats were significantly increased. In addition, the miR-145 expression level was upregulated, and the p-AKT protein expression level was decreased. The in vitro dual luciferase reporter assay demonstrated that miR-145 can bind with the 3'-UTR region of RREB1. These findings 
demonstrate that miR-145 can regulate RREB1 expression and affect the PI3K/AKT signaling pathway.

In conclusion, the results in the present study suggest that miR-145 serves an important role in the development of neuropathic pain through regulating RREB1 expression and the PI3K/AKT signaling pathway. In addition, the results indicate that miR-145 may be a potential target in the diagnosis and treatment of neuropathic pain.

\section{Acknowledgements}

The authors thank Dr. Jiaxiang Ni from the Department of Pain Management, Xuanwu Hospital of Capital Medical University, for his valuable help during the preparation of the manuscript.

\section{References}

1. Gilron I, Baron R and Jensen T: Neuropathic pain: Principles of diagnosis and treatment. Mayo Clin Proc 90: 532-545, 2015.

2. Fontaine D, Blond S, Mertens $\mathrm{P}$ and Lanteri-Minet M: Neurosurgical treatment of chronic pain. Neurochirurgie 61 : 22-29, 2015.

3. Fallon MT, Storey DJ, Krishan A, Weir CJ, Mitchell R, Fleetwood-Walker SM, Scott AC and Colvin LA: Cancer treatment-related neuropathic pain: Proof of concept study with menthol-a TRPM8 agonist. Support Care Cancer 23: 2769-2777, 2015.

4. Helfert SM, Reimer M, Höper J and Baron R: Individualized pharmacological treatment of neuropathic pain. Clin Pharmacol Ther 97: 135-142, 2015.

5. Rice AS and Smith MT: Angiotensin II type 2-receptor: New clinically validated target in the treatment of neuropathic pain. Clin Pharmacol Ther 97: 128-130, 2015.

6. Jamali Z, Asl Aminabadi N, Attaran R, Pournagiazar F, Ghertasi Oskouei S and Ahmadpour F: MicroRNAs as prognostic molecular signatures in human head and neck squamous cell carcinoma: A systematic review and meta-analysis. Oral Oncol 51: 321-331, 2015.

7. Bienertova-Vasku J, Novak $J$ and Vasku A: MicroRNAs in pulmonary arterial hypertension: Pathogenesis, diagnosis and treatment. J Am Soc Hypertens 9: 221-234, 2015.

8. Greco S, Gorospe M and Martelli F: Noncoding RNA in age-related cardiovascular diseases. J Mol Cell Cardiol 83: 142-155, 2015.

9. Saab BJ and Mansuy IM: Neuroepigenetics of memory formation and impairment: The role of microRNAs. Neuropharmacology 80 61-69, 2014.

10. Akerblom M and Jakobsson J: MicroRNAs as neuronal fate determinants. Neuroscientist 20: 235-242, 2013.
11. Lutz BM, Bekker A and Tao YX: Noncoding RNAs: New players in chronic pain. Anesthesiology 121: 409-417, 2014.

12. Guo X, Yu L, Chen M, Wu T, Peng X, Guo R and Zhang B: miR-145 mediated the role of aspirin in resisting VSMCs proliferation and anti-inflammation through CD40. J Transl Med 14:211, 2016

13. O'Leary L, Sevinç K, Papazoglou IM, Tildy B, Detillieux K, Halayko AJ, Chung KF, and Perry MM: Airway smooth muscle inflammation is regulated by microRNA-145 in COPD. FEBS Lett 590:1324-1334, 2016.

14. Lu A, Huang Z, Zhang C, Zhang X, Zhao J, Zhang H, Zhang Q, Wu S and Yi X: Differential expression of microRNAs in dorsal root ganglia after sciatic nerve injury. Neural Regen Res 9: 1031-1040, 2014.

15. Norcini M, Sideris A, Martin Hernandez LA, Zhang J, Blanck TJ and Recio-Pinto E: An approach to identify microRNAs involved in neuropathic pain following a peripheral nerve injury. Front Neurosci 8: 266, 2014.

16. Massi D, Cesinaro AM, Tomasini C, et al: Atypical Spitzoid melanocytic tumors: a morphological, mutational, and FISH analysis. J Am Acad Dermatol 64:919-935, 2011.

17. Franklin RB, Zou J and Costello LC: The cytotoxic role of RREB1, ZIP3 zinc transporter, and zinc in human pancreatic adenocarcinoma. Cancer Biol Ther 15:1431-1437, 2014.

18. Hargreaves KM: Capsicum and local anesthetic cocktails for trigeminal pain. Pain 150: 3, 2010.

19. Livak KJ and Schmittgen TD: Analysis of relative gene expression data using real-time quantitative PCR and the $2^{-\Delta \Delta C t}$ method. Methods 25: 402-408, 2001.

20. Gilron I, Baron R and Jensen T: Neuropathic pain: Principles of diagnosis and treatment. Mayo Clin Proc 90: 532-545, 2015.

21. Aksoy-Aksel A, Zampa F and Schratt G: MicroRNAs and synaptic plasticity-a mutual relationship. Philos Trans R Soc Lond B Biol Sci 369: 20130515, 2014

22. Zhang HY, Zheng SJ, Zhao JH, Zhao W, Zheng LF, Zhao D, Li JM, Zhang XF, Chen ZB and Yi XN: MicroRNAs 144, 145, and 214 are down-regulated in primary neurons responding to sciatic nerve transection. Brain Res 1383: 62-70, 2011.

23. Kent OA, Fox-Talbot K and Halushka MK: RREB1 repressed miR-143/145 modulates KRAS signaling through downregulation of multiple targets. Oncogene 32: 2576-2585, 2013.

24. Boufraqech M, Zhang L, Jain M, Patel D, Ellis R, Xiong Y, He M, Nilubol N, Merino MJ and Kebebew E: miR-145 suppresses thyroid cancer growth and metastasis and targets AKT3. Endocr Relat Cancer 21: 517-531, 2014.

25. Noguchi S, Yasui Y, Iwasaki J, Kumazaki M, Yamada N, Naito $S$ and Akao Y: Replacement treatment with microRNA-143 and-145 induces synergistic inhibition of the growth of human bladder cancer cells by regulating PI3K/Akt and MAPK signaling pathways. Cancer Lett 328: 353-361, 2013.

26. Stein AT, Ufret-Vincenty CA, Hua L, Santana LF and Gordon SE: Phosphoinositide 3-kinase binds to TRPV1 and mediates NGF-stimulated TRPV1 trafficking to the plasma membrane. J Gen Physiol 128: 509-522, 2006. 